patients without additional excision (group 1; $n=199$ ); patients with re-excision found to be free of cancer (group 2; $n=546$ ); and patients with re-excision and residual cancer or carcinoma in situ (group 3; $n=299$ ). There was no significant difference in 10-year local control rate between women who did not undergo re-excision (group 1) and those who did (groups 2 and 3; 95\% vs 94\%, respectively; $P=0.788$ ). Of those who underwent re-excision, only $35 \%$ had residual disease. Initial positive margins, positive nodes, stage T2 tumor, and an extensive intraductal component were significantly associated with positive findings on re-excision. The 10-year local control rate was significantly lower for women with residual tumor than for those without (91\% vs 95\%; $P=0.038)$. Tenyear overall survival rates for groups 1, 2 and 3 were $70 \%, 79 \%$ and $73 \%$, respectively.

Given the low recurrence rates seen in this study, the authors suggest that selected patients with non-negative margins may be treated with radiotherapy alone. The need for re-excision should be carefully weighed against the likelihood of residual disease, the cosmetic effect on the breast and the degree of risk reduction it might afford.

Original article Chism DB et al. (2006) Re-excision of margins before breast radiation-diagnostic or therapeutic? Int J Radiat Oncol Biol Phys 65: 1416-1421

\section{FDG-PET: a powerful predictor of survival following chemotherapy in advanced breast cancer}

A single ${ }^{18} \mathrm{~F}$-fluorodeoxyglucose (FDG)-PET scan performed following high-dose chemotherapy (HDC) is a powerful independent predictor of survival in patients with metastatic breast cancer (MBC), according to Cachin et al.'s recent study.

In this study, 47 patients with $\mathrm{MBC}$ received a maximum of three consecutive cycles of HDC with autologous stem cell transplantation. Therapeutic response was assessed 1 month after the last cycle of HDC using a range of conventional imaging techniques (Clmg; including CT and ultrasound) and FDG-PET. Overall, complete responses following HDC were seen in $37 \%$ of patients using Clmg and $72 \%$ using FDG-PET. At a median follow-up of 87 months, analysis showed that the FDG-PET result was the most powerful independent predictor of survival. Patients with a negative post-treatment FDG-PET result had a significantly longer median survival than those with a positive FDGPET result (24 months vs 10 months; $P<0.001$ ), and FDG-PET positivity was associated with a fivefold increased risk of death. Other factors associated with increased risk of death on multivariate analysis were prior anthracycline treatment (relative risk 3.3) and visceral metastasis (relative risk 2.4). Therapeutic response according to Clmg did not predict for significantly improved long-term survival.

The authors call for larger prospective studies of FDG-PET in patients undergoing conventional-dose therapy for MBC.

\section{Original article Cachin F et al. (2006) Powerful prognostic stratification by $\left[{ }^{18} \mathrm{~F}\right]$ fluorodeoxyglucose positron emission tomography in patients with metastatic breast cancer treated with high-dose chemotherapy. J Clin Oncol 24: 3026-3031 \\ Continued proliferation despite letrozole or tamoxifen in HER2-positive breast cancer}

Estrogen-receptor-positive breast cancers that overexpress HER2 show diminished response to tamoxifen. Data from small studies, however, suggest that response to neoadjuvant therapy with agents such as letrozole may not be impeded by HER2 overexpression.

To investigate the relationship between HER2 amplification and the efficacy of neoadjuvant endocrine therapy, Ellis and co-workers used fluorescence in situ hybridization (FISH) to detect HER1 and HER2 gene expression in tissue samples from 305 postmenopausal women with stage II and III estrogen-receptor-positive breast cancer. They then correlated HER2 FISH status with response and changes in the proliferation marker Ki67 following treatment with neoadjuvant letrozole or tamoxifen. They found that whereas HER1 amplification was present in fewer than $1 \%$ of tumors, HER2 amplification was seen in $9.2 \%$ of tumors and was associated with significantly higher histological grade $(P=0.009)$ and pretreatment Ki67 level $(P=0.005)$ than HER2 FISH-negative tumors. HER2 FISH-positivity did not affect letrozole response as assessed by mammogram or ultrasound, but there was significantly less Ki67 suppression following letrozole administration in these tumors $(P=0.0001)$. Similar results were seen in tamoxifen-treated patients, although the 